\title{
Search for Exotic Physics Beyond the Standard Model with the ATLAS Detector
}

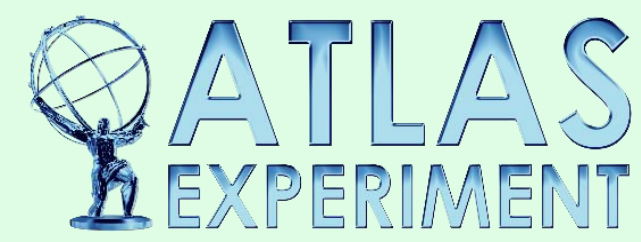

\author{
Yury Smirnov \\ (NRNU MEPhI, Moscow) \\ on behalf of the \\ ATLAS Collaboration
}

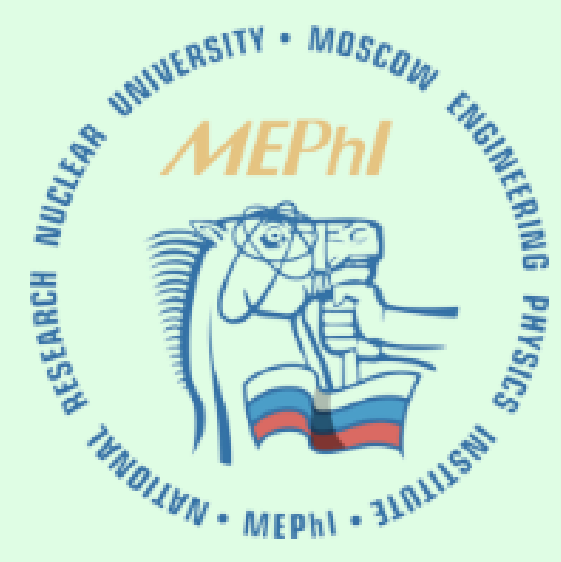

$1^{\text {th }}$ Lomonosov Conference on Elementary Particle Physics 20-26 August 2015 


\section{Outline: ATLAS searches for Exotic physics}

> Very extensive program: about 50 searches with full $2012(\sqrt{s}=8 \mathrm{TeV})$ dataset;

$>$ Dibosons, multileptons, extra dimensions, heavy quarks, top, composite Higgs, jets + dark matter, unconventional signatures, exotic Higgs, W', Z', lepton flavor violation, ...;

$>$ Only the following searches are covered in this talk:

Photon+missing $\mathrm{E}_{\mathrm{T}}$

Heavy Majorana neutrino

$$
Z^{\prime} \rightarrow \tau^{+} \tau^{-}
$$

Long-lived neutral particles $\rightarrow$ displaced lepton jets

$$
\begin{gathered}
X \rightarrow t \bar{t} \rightarrow W^{+} b W^{-} \bar{b} \\
H \rightarrow Z_{\text {dark }} Z_{[\text {dark }]} \rightarrow 4 l^{ \pm}
\end{gathered}
$$

High-mass diphoton resonances

Multi-charged particles

Low-scale gravity signatures
Searches with slight excesses in Run-I data:

Events with $b$-jets and pair of same-sign leptons

$$
X \rightarrow W W / W Z / Z Z \rightarrow j j
$$

Several preliminary plots from early Run-II

$$
\text { searches }
$$




\section{ATLAS detector}

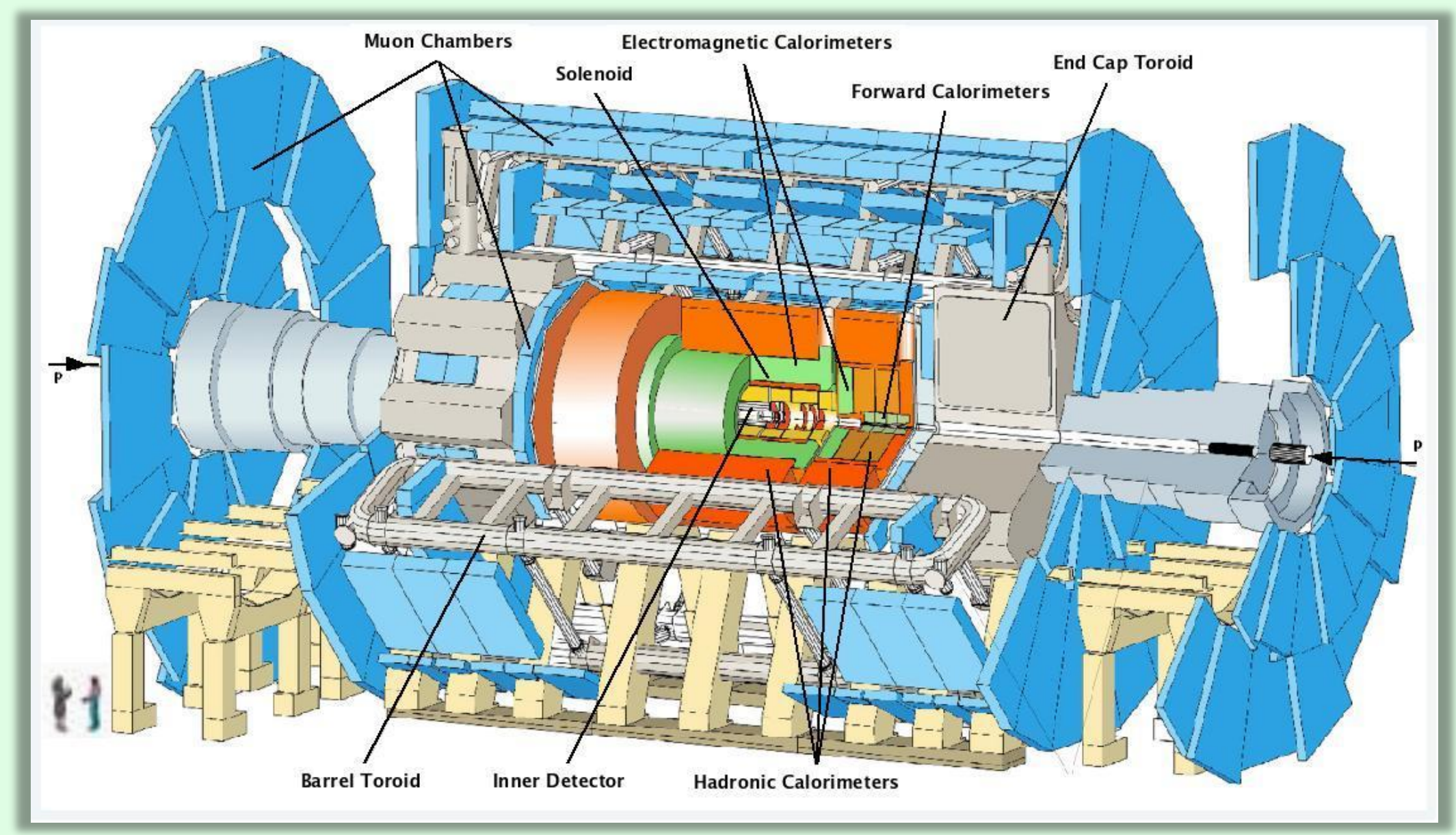

- General-purpose detector with near $4 \pi$ coverage in solid angle;

- Inner tracking detector, calorimeters system, muon spectrometer;

- Physics with final states: $e^{ \pm}, \mu^{ \pm}$, jets, $\gamma$, missing transverse momentum $\left(\mathbb{Z}_{T}\right), \ldots$ 


\section{$\mathrm{Y}+\mathrm{MET}$ search}

$>$ Search for events with a high- $p_{T}$ photon and large $\mathbb{Z}_{T}$ with no $e^{ \pm}$or $\mu^{ \pm}$;

$>$ Main background source is $Z(\rightarrow v \bar{v})+\gamma_{I S R}$

$>$ Results are interpreted in terms of limits on the parameters of large extra dimension theories, WIMP dark matter and supersymmetric quarks.
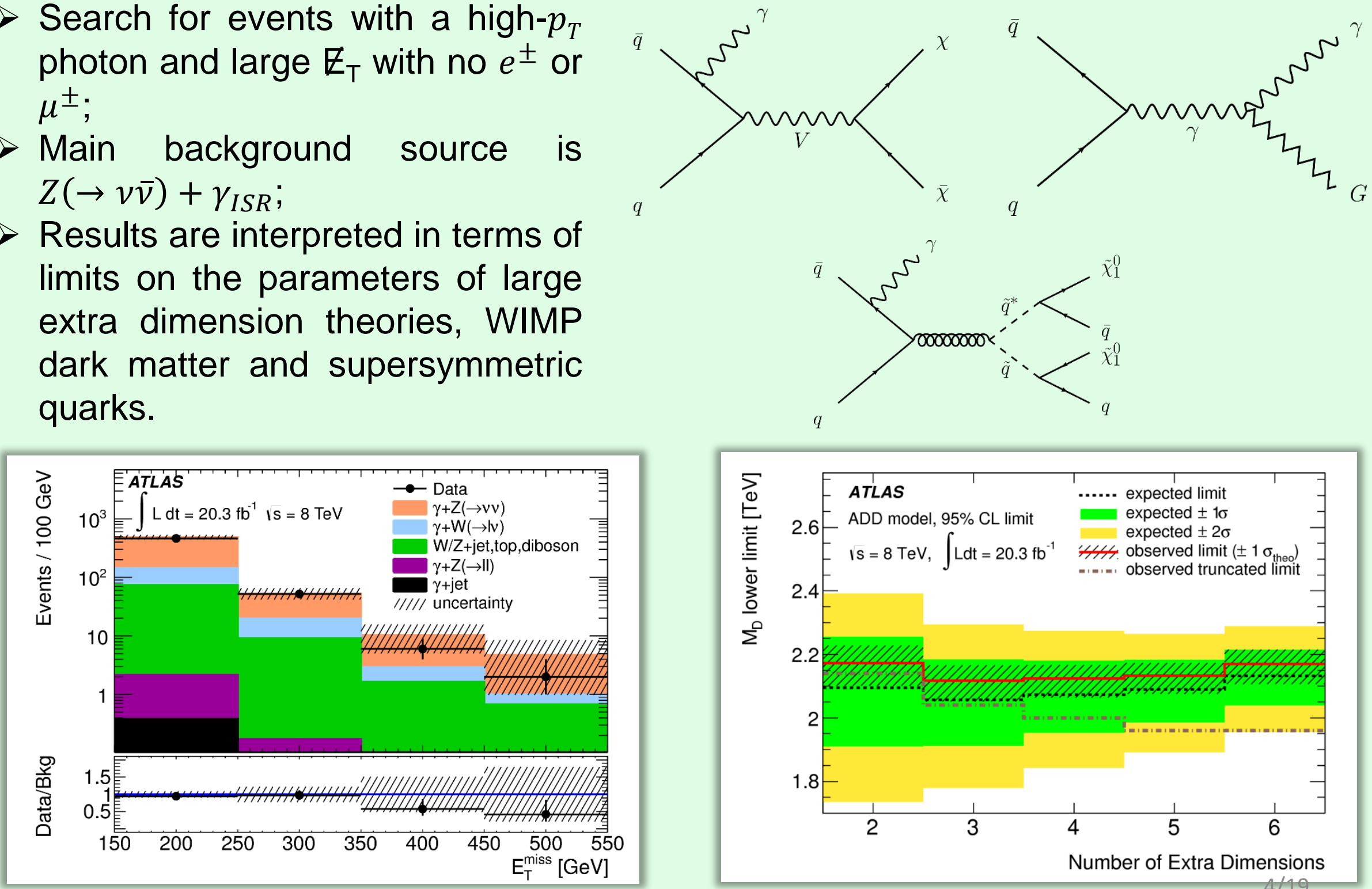

Yury Smirnov 


\section{$Z^{\prime} \rightarrow \tau^{+} \tau^{-}$search}

$>$ Two final state channels: $\tau^{ \pm} \rightarrow l^{ \pm} v \bar{v}$ (leptonic channel, $\tau_{\text {lep }}$ ) and hadronic channel $\left(\tau_{\text {had }}\right)$;

$>$ For both channels, the dominant background source is $Z / \gamma^{*} \rightarrow \tau \tau$, estimated from simulation and validated using $Z / \gamma^{*}$ decays to electrons and muons in data;

$>$ Limits on the $Z^{\prime}$ mass for the Sequential Standard Model and non-universal $G(221)$ model.
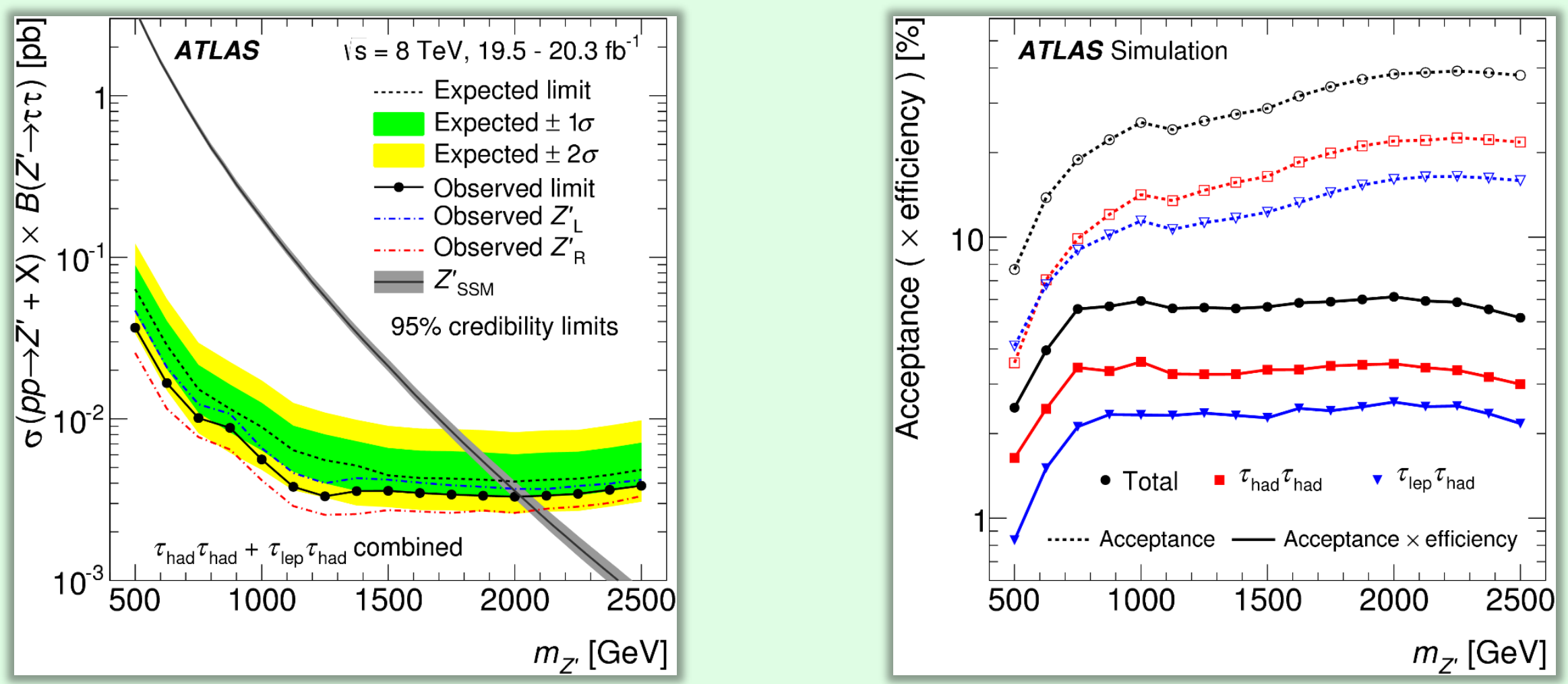

$6 / 19$ 


\section{Search for long-lived neutral \\ arXiv:1409.0746; \\ JHEP 11 (2014) 088 \\ particles decaying into displaced lepton jets (LJs)}

$>$ Unstable hidden states $\rightarrow$ hidden particles $\left(\gamma_{\text {dark }}\right)$ with nonnegligible lifetime due to their weak interaction $\rightarrow$ visible final states (collimated jet-like
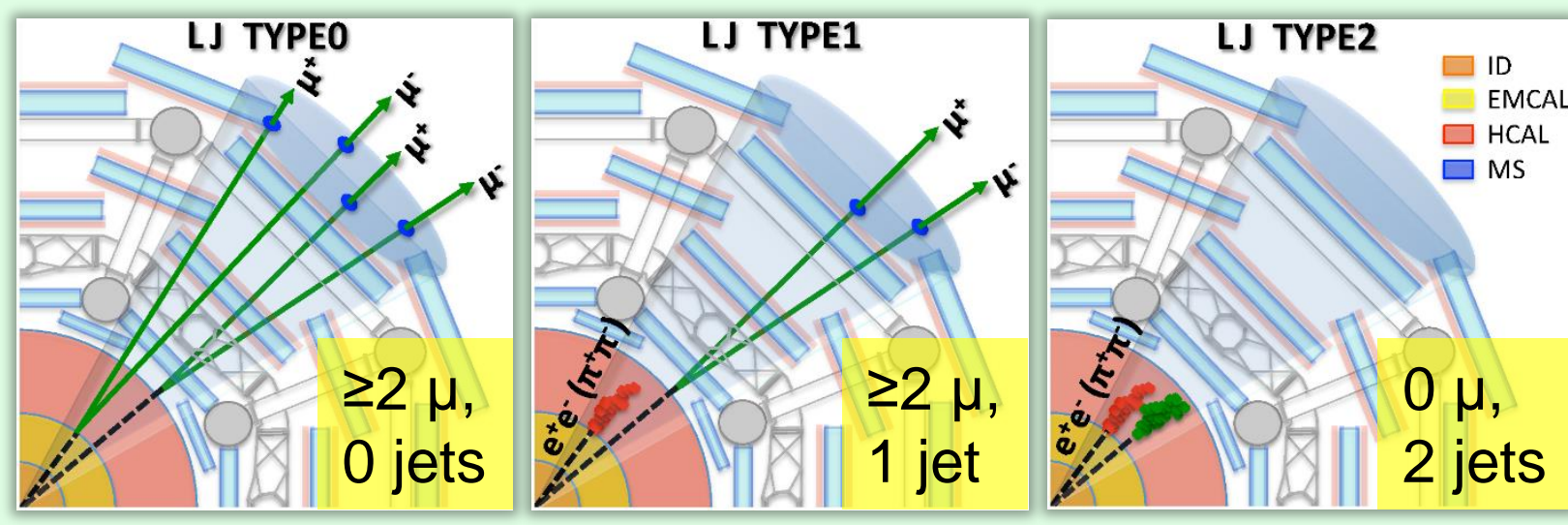
structures containing pairs of $e^{ \pm}$or $\mu^{ \pm}$or $\pi^{ \pm}$);

$>$ Background:

- SM processes $\mathrm{w} /$ or w/o jets: $W+$ jets, $Z+$ jets, $t \bar{t}, \ldots \Rightarrow$ data-driven matrix method;

- Cosmic-ray muons + detector material $\rightarrow$ background to LJ TYPE1 and TYPE2 $\Rightarrow$ empty bunches analysis;

$>$ Limits on $\sigma \times B R$ for non-SM Higgs boson decays to dark photons and LJs (FRVZ model); limits on kinetic mixing parameter $\epsilon$ as $f\left(m_{\gamma_{\text {dark }}}\right)$.

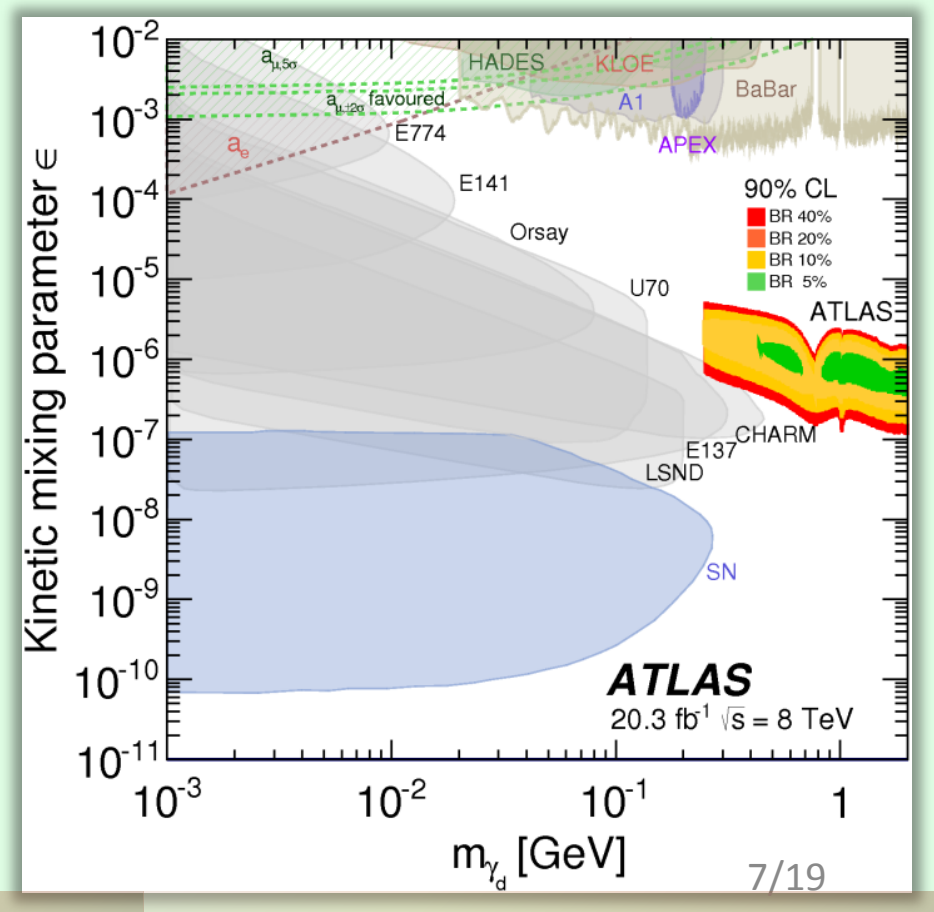




\section{$X \rightarrow t \bar{t} \rightarrow W^{+} b W^{-} \bar{b}$ search}

arXiv:1505.07018; accepted by JHEP

> Top quarks: the most massive SM fundamental particles, can have large coupling to heavy Higgs bosons;

> General search; composite Higgs and topcolourassisted technicolor scenarios propose mechanisms for EWSB, incorporating $X$ with large coupling to $t \bar{t}$ (w.r.t. lighter quarks);

> One W boson decays leptonically, the other one decays hadronically $\Rightarrow$ "lepton-plus-jets events";

$>$ Events signature: 1 isolated $e^{ \pm} / \mu^{ \pm}, \mathbb{Z}_{\mathrm{T}}$, hadronic jets consistent with having originated from $a b$ quark;

$>$ Background to the $m_{t \bar{t}}$ spectrum: $W+$ jets and $t \bar{t}$ processes $\Rightarrow$ data- and MC-driven estimations;

> Results are interpreted as mass limits on the $Z^{\prime}$, heavy Kaluza-Klein gluons, Kaluza-Klein excitations of the graviton, and color-singlet scalar particles.
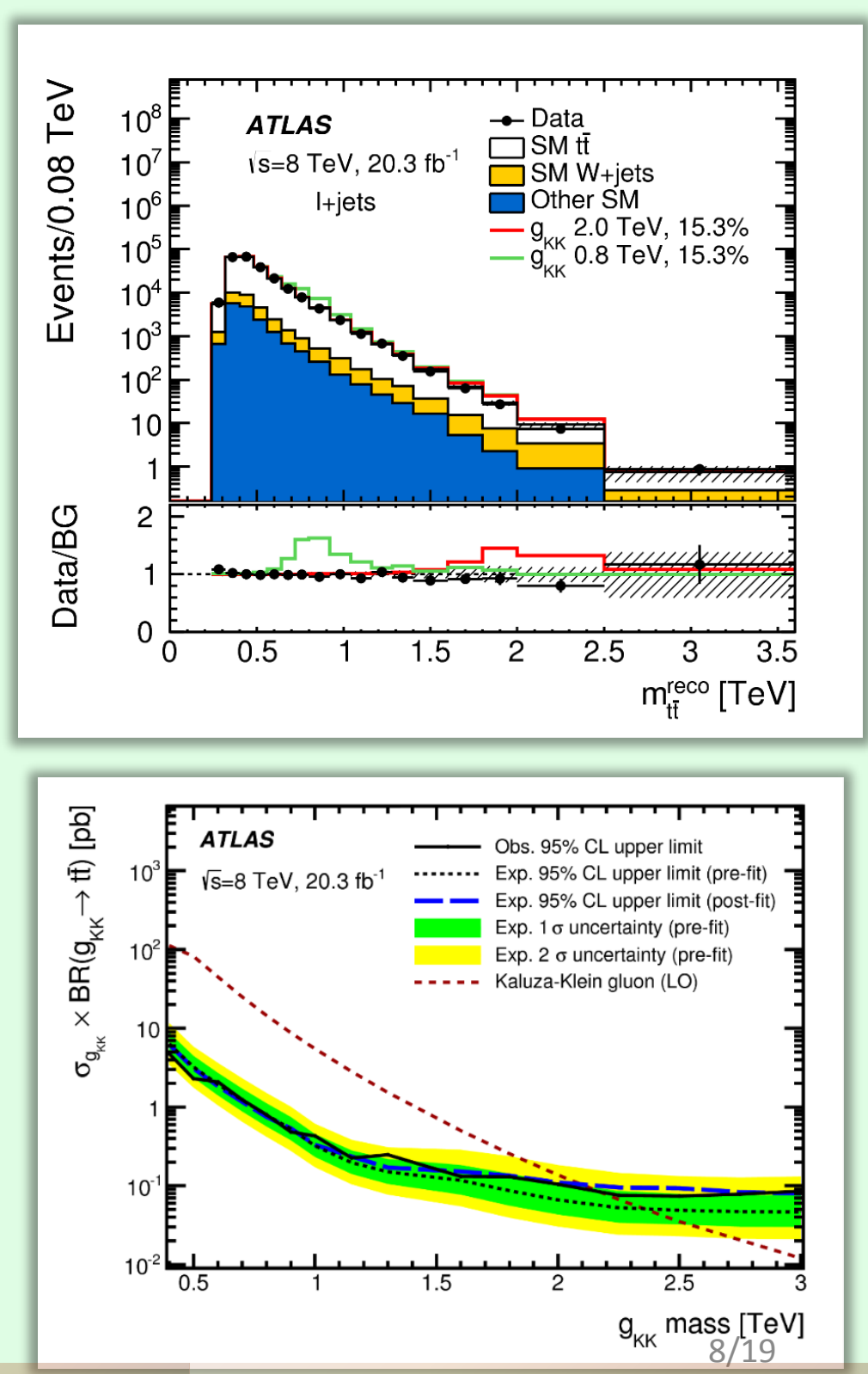


\section{Search for new light gauge bosons axxiv:1505.07645; Search for new light gauge bosons submitted to PRD}

\section{in $H \rightarrow Z_{\text {dark }} Z_{[\text {dark }]} \rightarrow 4 l^{ \pm}\left(e^{ \pm}\right.$or $\left.\mu^{ \pm}\right)$}

$>H \rightarrow Z_{\text {dark }} Z$ search: test for excess an invariant mass spectrum of two leptons, not associated with $Z$; backgrounds are $Z Z^{*}, t \bar{t}$ and $Z+$ jets $\Rightarrow$ datadriven methods;

$>H \rightarrow Z_{\text {dark }} Z_{\text {dark }}$ search: look for a small difference in invariant masses of pairs of two same-flavor opposite-charge leptons; backgrounds are $\mathrm{H} \rightarrow$ $Z Z^{*} \rightarrow 4 l, Z Z^{*} \rightarrow 4 l, W W, W Z, t \bar{t}$ and $Z+$ jets processes $\Rightarrow$ estimated from simulation;

$>$ Interpretation in terms of limits on $\frac{B R\left(H \rightarrow Z_{\text {dark }} Z \rightarrow 4 l^{ \pm}\right)}{B R\left(H \rightarrow Z Z^{*} \rightarrow 4 l^{ \pm}\right)}$ vs. $m_{Z_{d}}$ and $95 \% \mathrm{CL}$ upper bound on the branching ratio of $H \rightarrow Z_{\text {dark }} Z_{\text {dark }}$ in the combined $4 e+2 e 2 \mu+4 \mu$ final state;

$>H \rightarrow Z_{\text {dark }} Z_{\text {dark }}$ search: no significant excess, but two events did pass tight requirements.
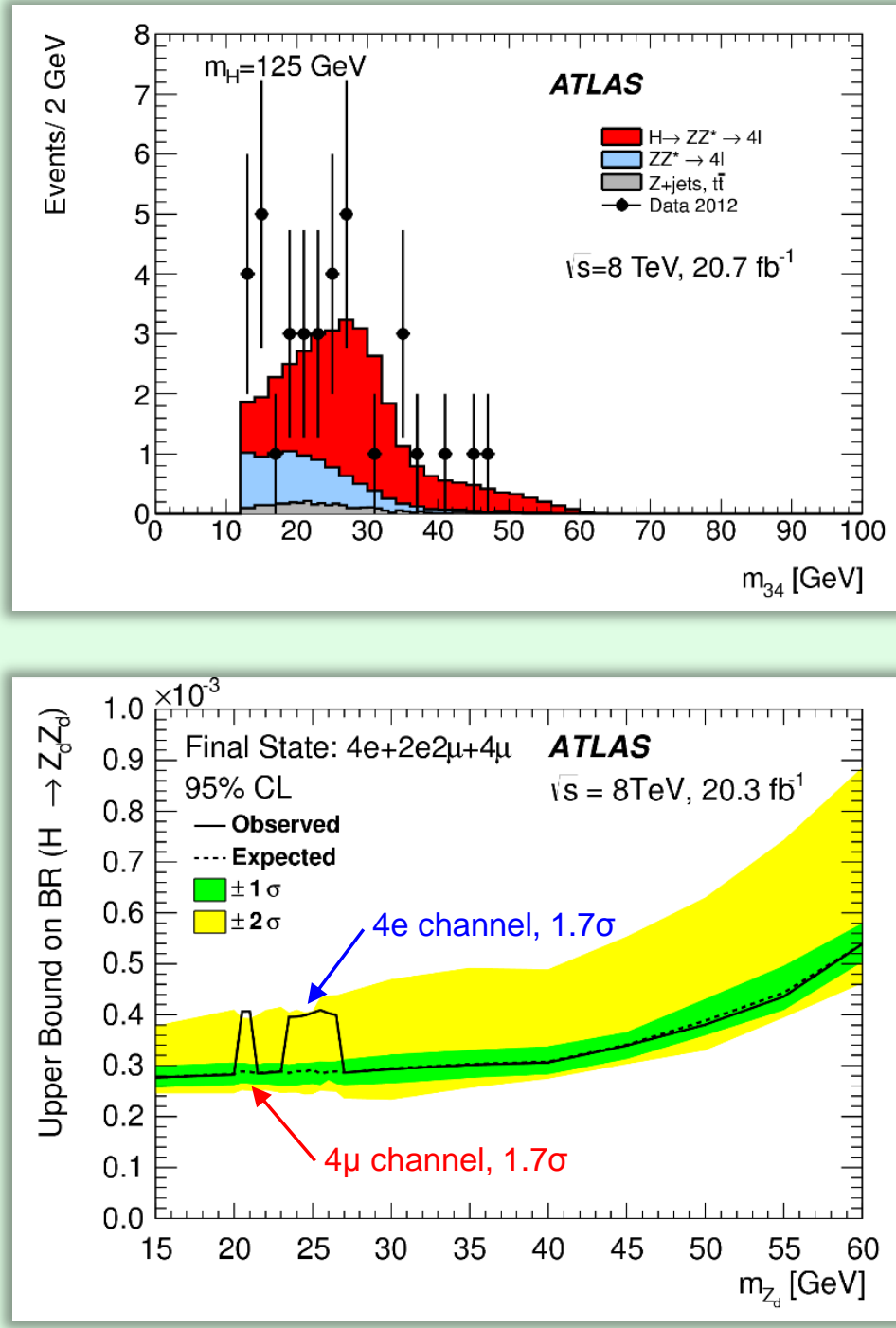

$9 / 19$ 


\section{Search for high-mass diphoton resonances}

$>$ Randall-Sundrum model: solving the SM hierarchy problem assuming an extra spatial dimension; its compactification leads to a Kaluza-Klein tower of gravitons excitations $G^{*}$;

$>$ Model phenomenology: the mass of the lightest KK graviton excitation $m_{G^{*}}$ and the dimensionless coupling to the SM fields $k / \bar{M}_{P l}$;

$>$ Diphoton trigger and two isolated high- $\mathrm{E}_{\mathrm{T}}$ photons;

$>$ Dominant background is SM processes with prompt $\gamma \gamma$ production: events with $\gamma+$ jet or jet+jet, where one or two jets are reconstructed as photons. Irreducible bkg contribution is obtained from $\mathrm{MC}$, a reducible one - from data;
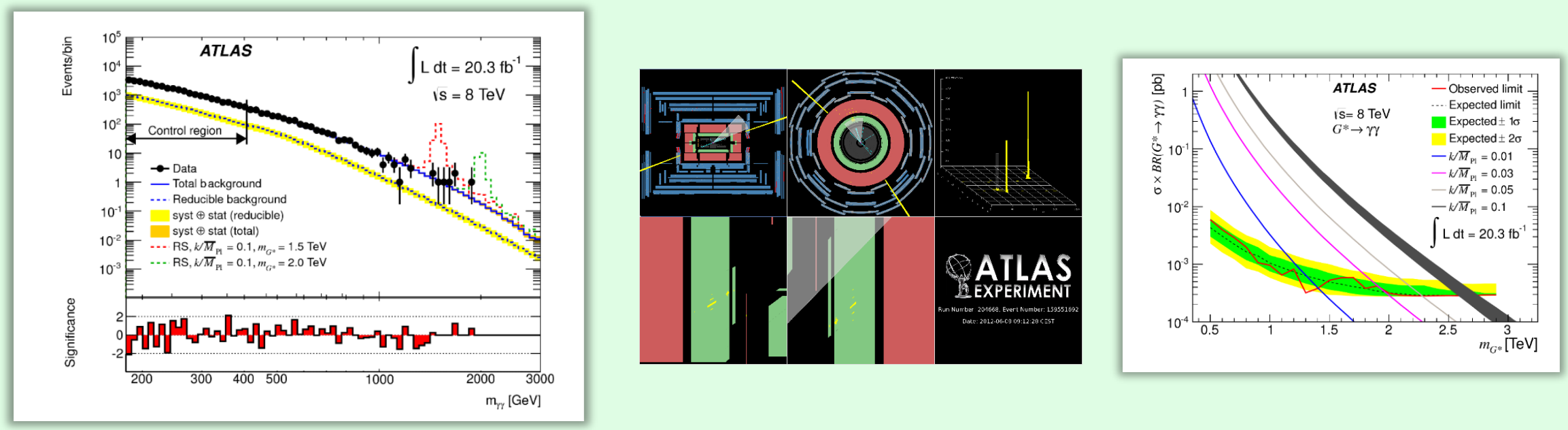

$>$ Limits on $\sigma \times B R\left(G^{*} \rightarrow \gamma \gamma\right)$ and $m_{G^{*}}$. 


\section{Search for multi-charaed particles arkiv:1504.04188;}

$>$ "Blue-sky" search for heavy long-lived particles with $2 \leq|q| / e \leq 6$;

$>$ Events with at least one muon-like particle with high ionization loss along its trajectory;

$>$ Main background: high- $p_{T}$ muons $\Rightarrow$ estimated with data-driven matrix method;

$>$ Limits on $\sigma$ and mass as a function of charge.
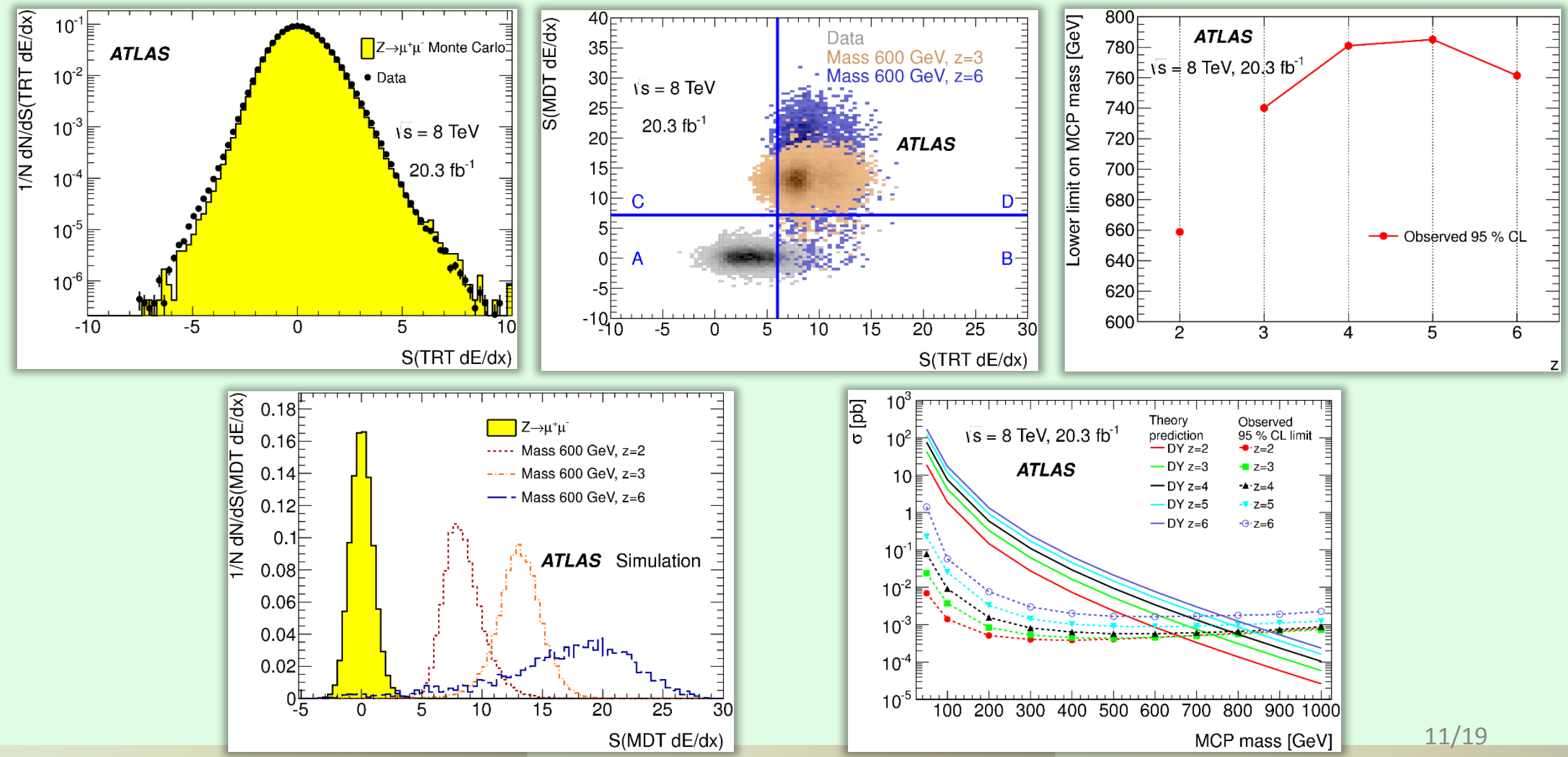

Yury Smirnov

August 20-26 2015

$11 / 19$

Lomonosov-2015 


\section{Search for low-scale gravity}

arXiv:1503.08988; signatures

$>$ Quantum gravity $\rightarrow$ hierarchy problem $\rightarrow$ existence of non-perturbative gravitational states: micro black holes, string balls, higherdimensional branes;

$>$ Events with multiple high- $p_{T}$ jets: scalar sum of $p_{T}$ of the jets in the event $H_{T}>1.5 \mathrm{TeV} \&$ $N_{\text {jet }} \geq 3$;

> Background: small contribution from SM QCD processes like $t \bar{t}, \gamma / W / Z+$ jets $\Rightarrow$ fit-based data-driven technique;

> Upper limits on visible cross-section for different jet multiplicities and lower mass limits on black holes and string balls masses.
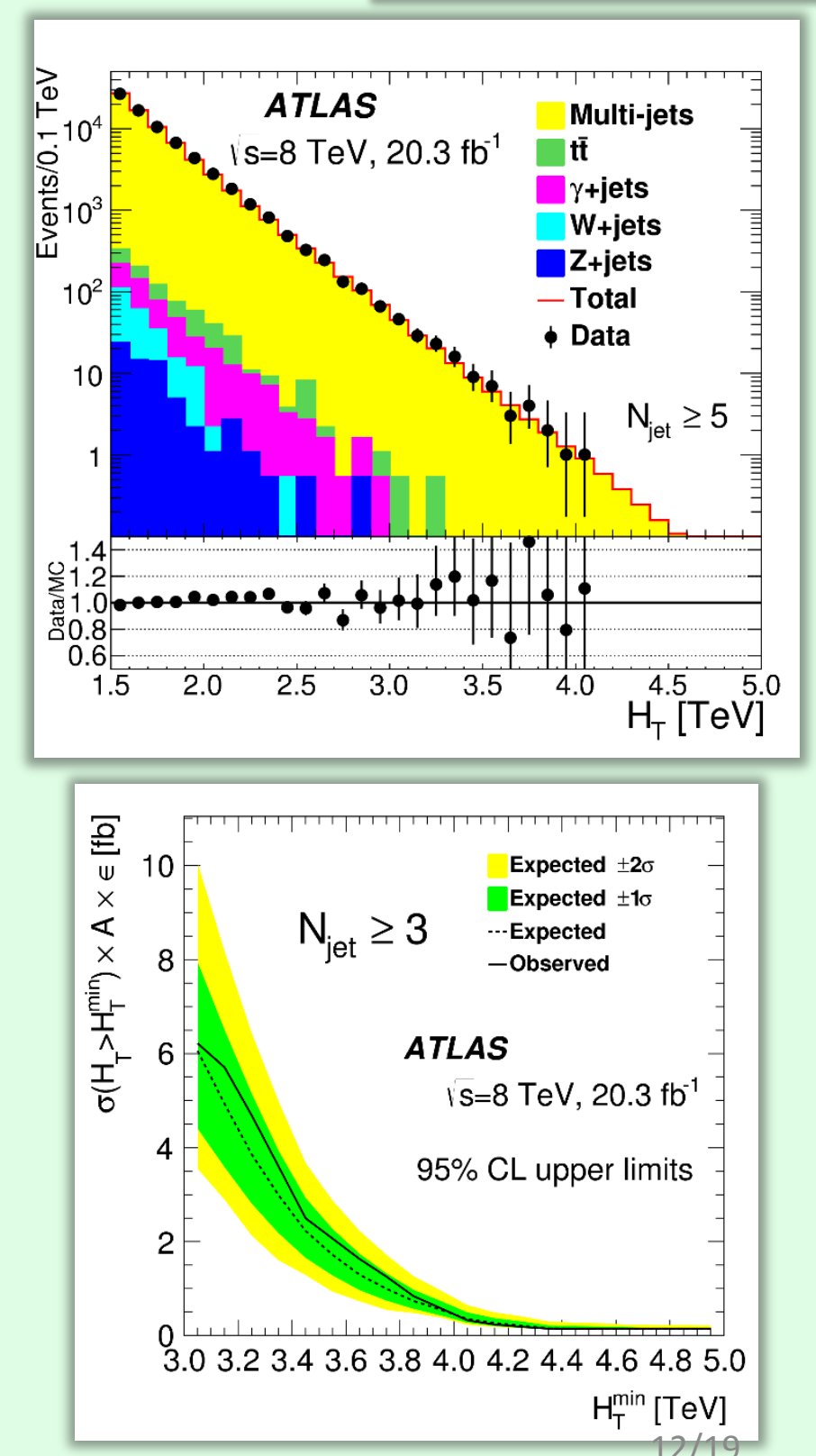


\section{Analysis of events with b-jets
pair of same-sign leptons}

$>$ Sensitive to models postulating vector-like quarks existence, enhancement of $t \bar{t} t \bar{t}$ production cross-section, chiral $4^{\text {th }}$ generation quarks existence, $t t$ production;

$>t \bar{t} t \bar{t}$ cross-section enhancement: via sgluon pair production \& pair production of Kaluza-Klein excitations of the photon decaying to $t \bar{t}$;

$>\geq 2$ same-sign leptons, $\geq 2$ jets (at least one should originate from a b-quark) and large $Z_{T}$;

$>$ Background: small SM yields from $W^{ \pm} W^{ \pm} j j, t \bar{t} W / Z, t \bar{t} W^{+} W^{-}, t \bar{t} H, W / Z H, \ldots+$ instrumental $\Rightarrow$ data-driven methods.
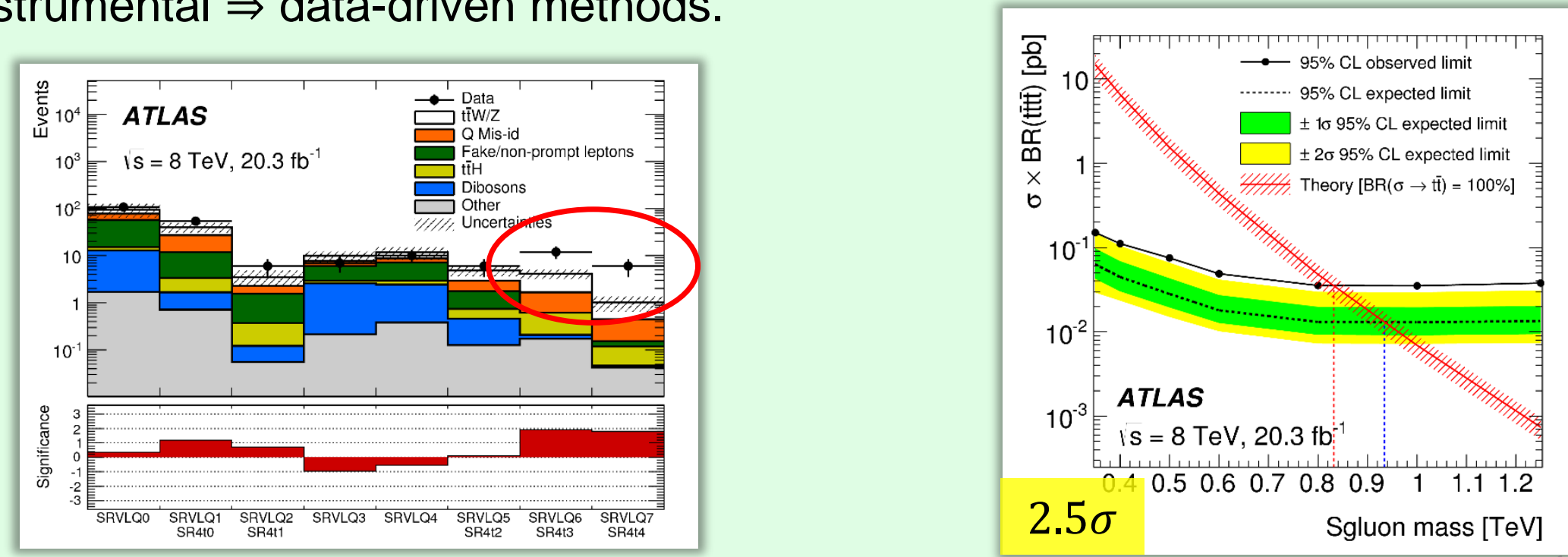


\section{First look at Run-II data}

\section{Run-II ( $\sqrt{s}=13 \mathrm{TeV}$ data) is here!}

$>$ No complete analyses available yet, some representative plots instead.

Dimuon invariant mass (more info)

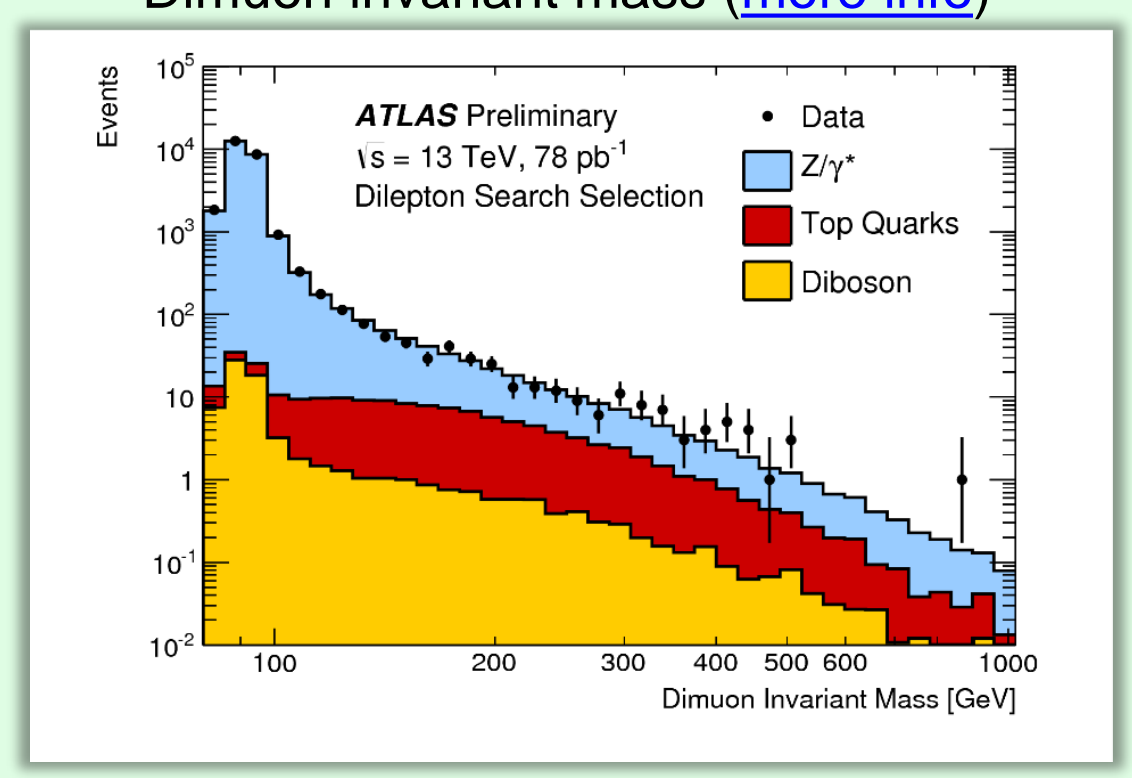

Corresponding Run-I result: arXiv:1405.4123
Diphoton invariant mass (more info)

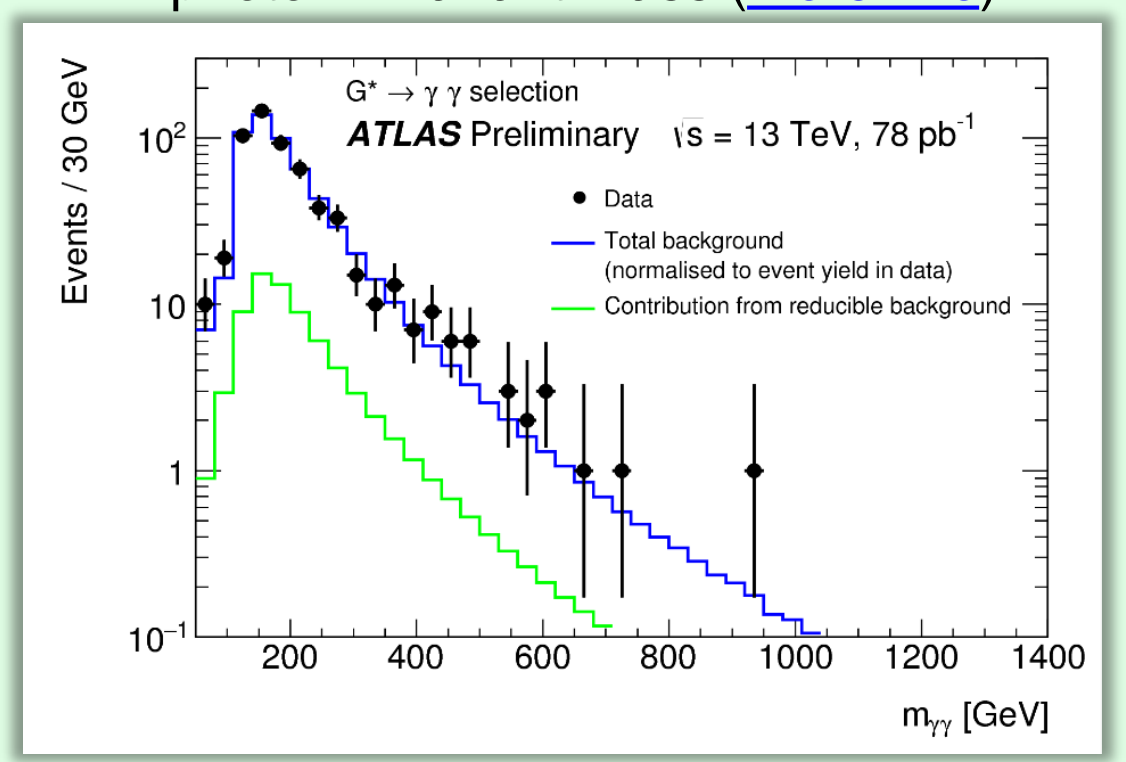

Corresponding Run-I result: arXiv:1504.05511 


\section{First look at Run-II data}

e- $\mu$ invariant mass $\left(Z^{\prime} \rightarrow e \mu\right)$ (more info)

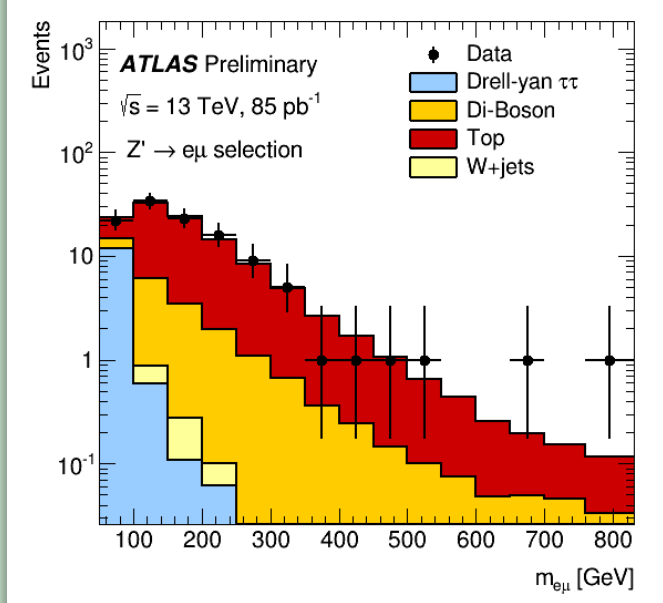

Corresponding Run-I result: arXiv:1503.04430
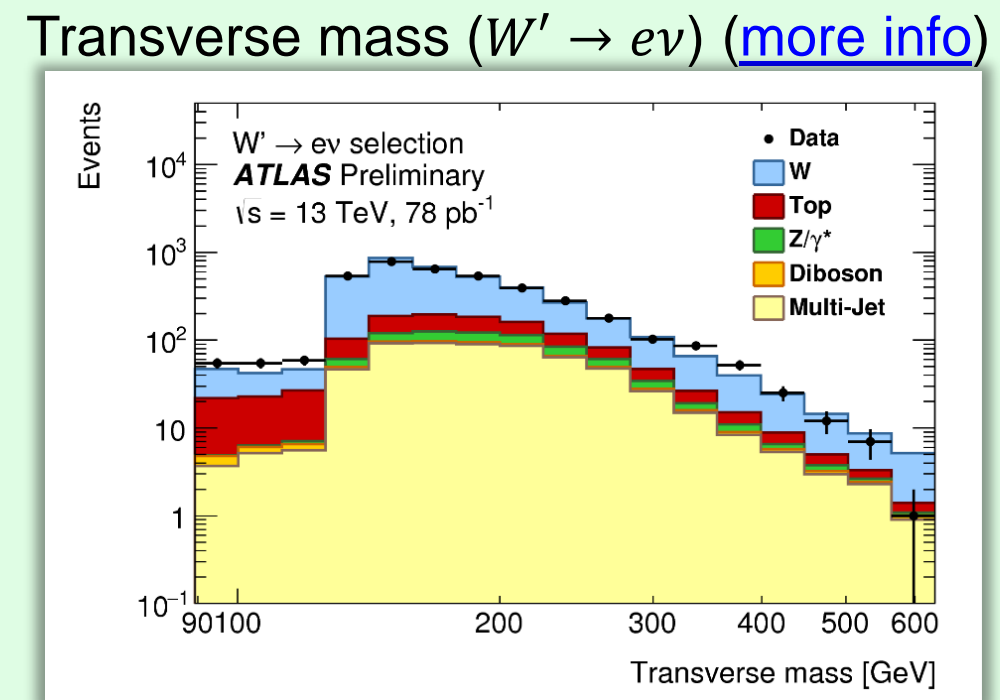

Corresponding Run-I result: arXiv:1407.7494 Yury Smirnov
Monojets: $\mathbb{Z}_{\mathrm{T}}$ in the $W \rightarrow \mu \nu \mathrm{CR}$ (more info)

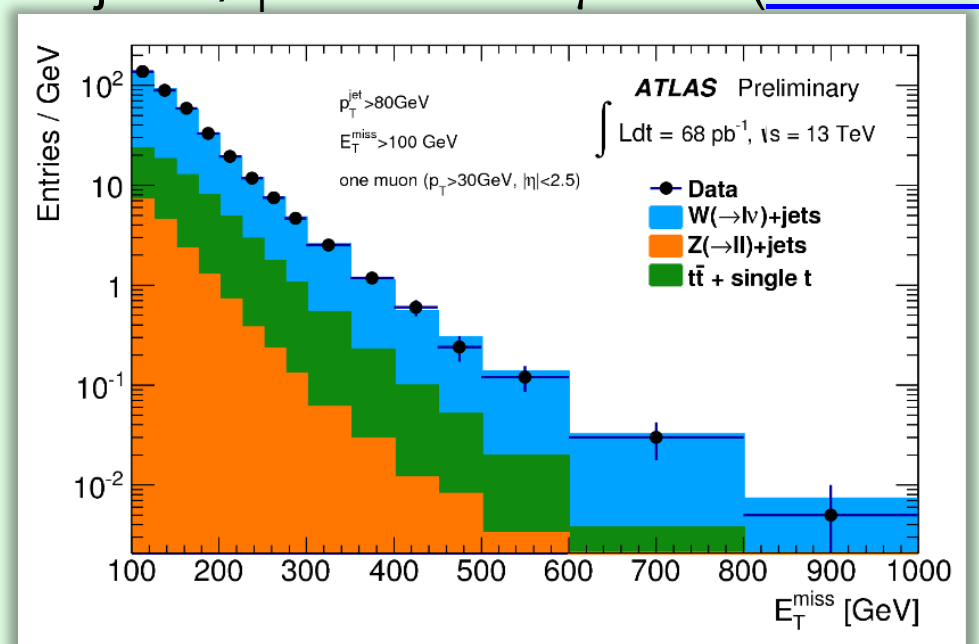

Corresponding Run-I result: arXiv:1407.0608

Mono- $\boldsymbol{b}: \mathbb{Z}_{\mathrm{T}}$ in the $1 \mu 0 e \mathrm{CR}$ (more info)

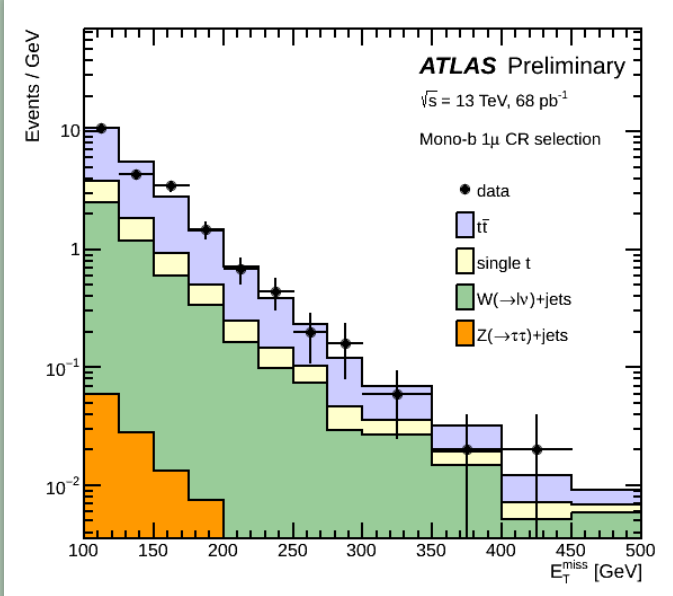

Corresponding Run-I result: arXiv:1410.403116/19 August $20-262015$ 


\section{... and much more}
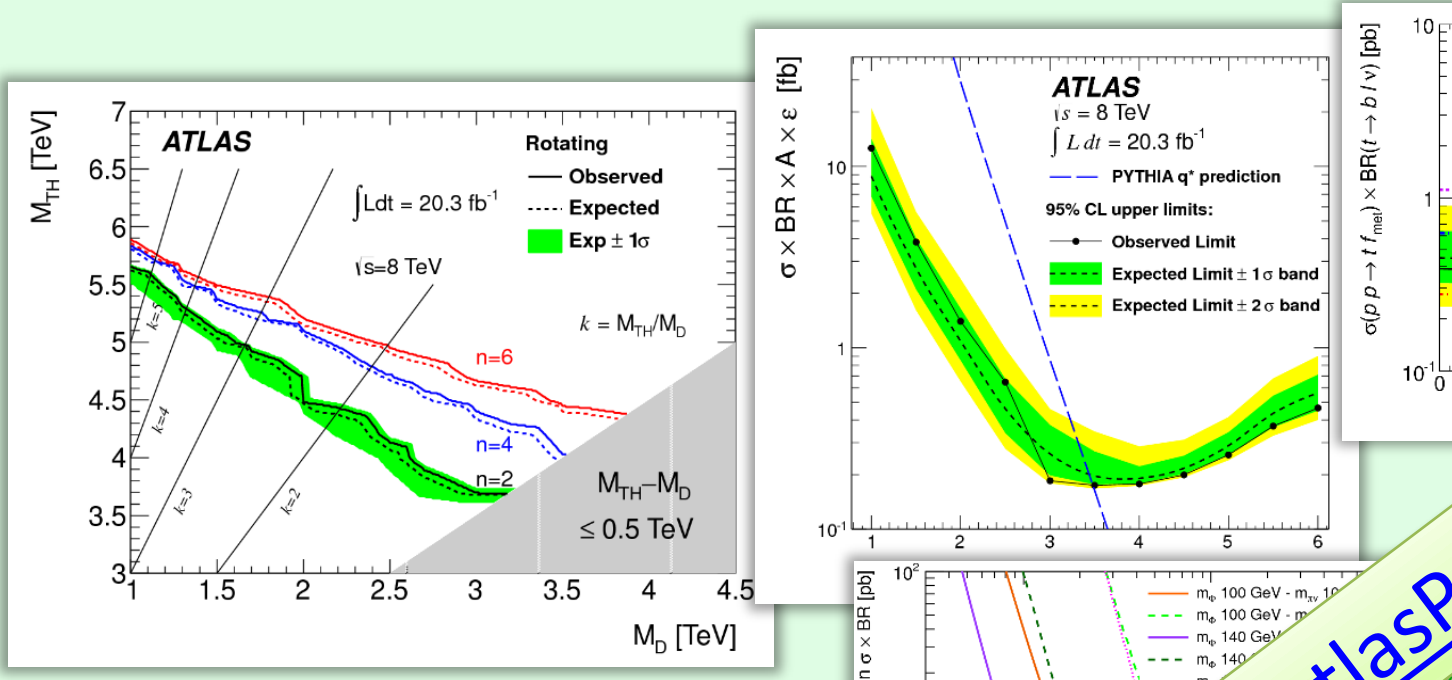

ATLAS ATLAS

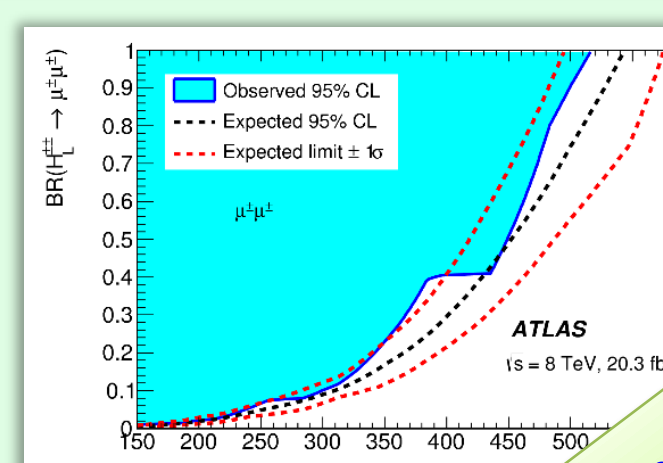

$-m_{\phi} 100 \mathrm{GeV}-m_{0}$
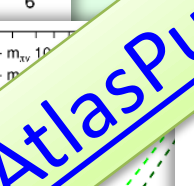

is $=8 \mathrm{TeV}, 20.3 \mathrm{fb}^{-1}, \mathrm{e}^{ \pm} / \mu^{ \pm} \cdots$.... Theory (LO), $a_{\mathrm{res}}=0.15$

Resonant model

$m(S)=500 \mathrm{GeV}$

Observed $95 \%$ CL limit
$\cdots-$ Expected $95 \%$ CL limit
$+1 \sigma$

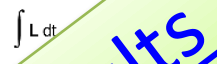

$\int 1 \mathrm{~d} / \mathrm{5}$
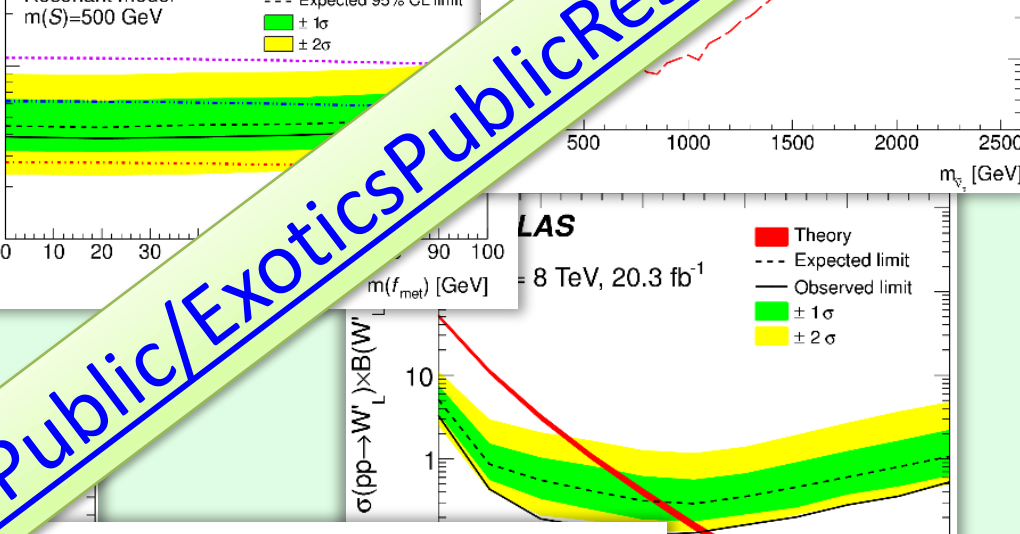

LAS

$8 \mathrm{TeV}, 20.3 \mathrm{fb}^{-1}$

Theory

- Expected limit

- Observed limit
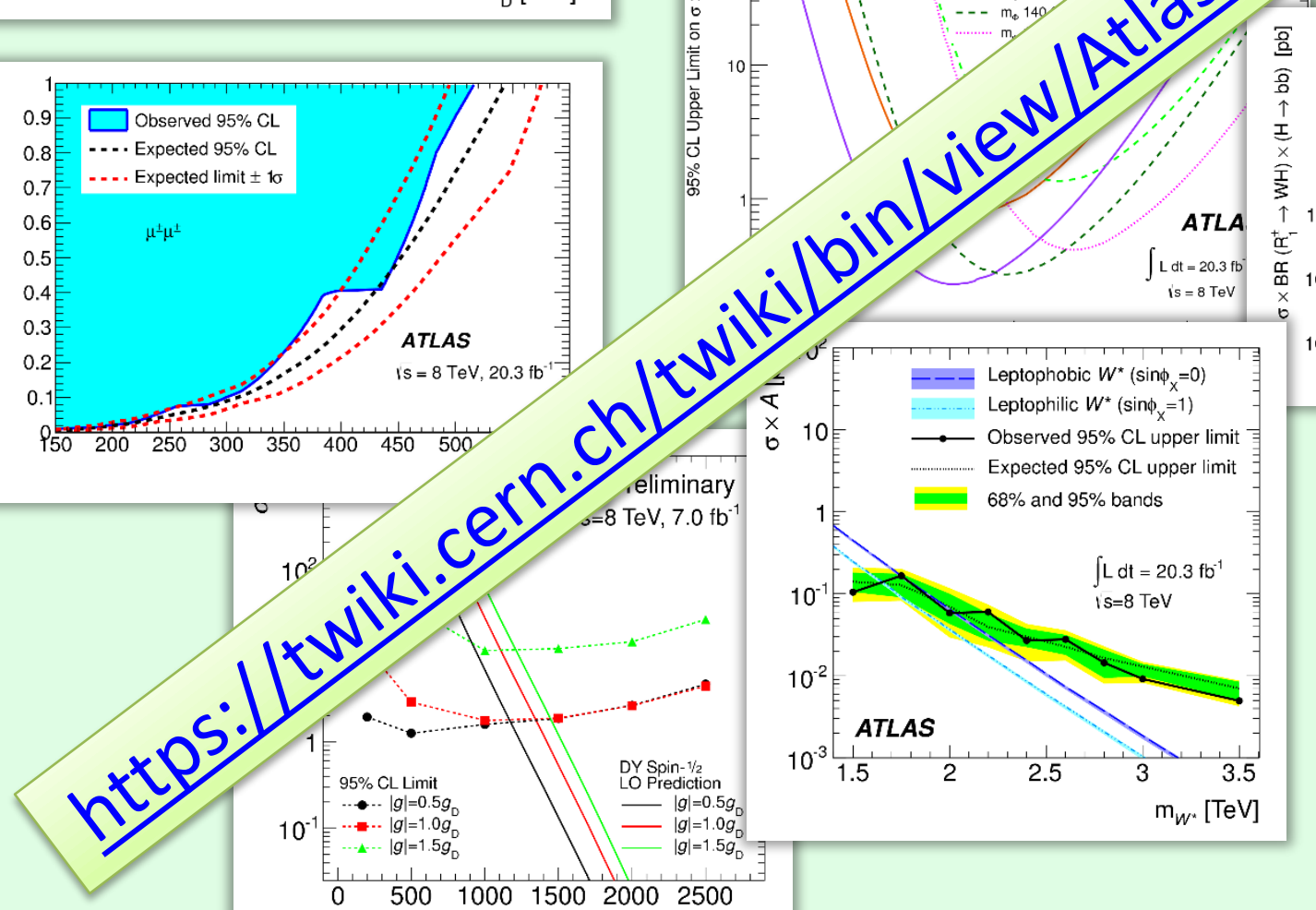

$\begin{array}{lllll}0 & 500 & 1000 & 1500 & 2000 \\ 2500\end{array}$
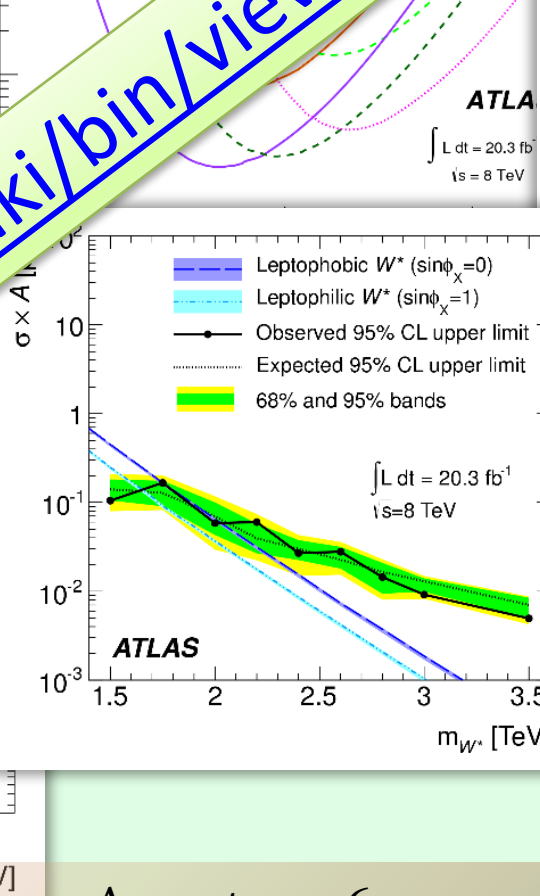

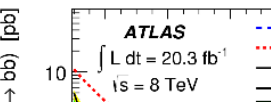
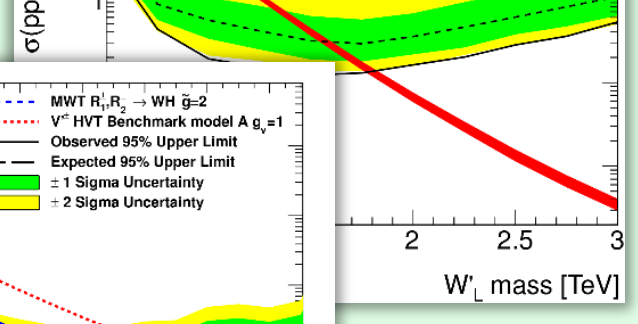

Yury Smirnov

$m[\mathrm{GeV}]$

August 20-26 2015

Lomonosov-2015 


\section{Exotics "mass reach" summary plot (as of July 2015), ...}

\section{ATLAS Exotics Searches* - 95\% CL Exclusion}

Status: July 2015

\section{Model}

\begin{tabular}{|c|c|}
\hline & $\begin{array}{l}\mathrm{ADD} G_{K K}+g / q \\
\mathrm{ADD} \text { non-resonant } \ell \ell\end{array}$ \\
\hline & $\mathrm{ADD} Q \mathrm{QBH} \rightarrow \ell q$ \\
\hline & ADD QBH \\
\hline & ADD BH high $N_{\text {trk }}$ \\
\hline & ADD BH high multijet \\
\hline & RS1 $G_{K K} \rightarrow \ell \ell$ \\
\hline 2ู & RS1 $G_{K K} \rightarrow \gamma \gamma$ \\
\hline 齐 & $\begin{array}{l}\text { Bulk RS } G_{K K} \rightarrow Z Z \rightarrow q q \ell \ell \\
\text { Bulk RS } G_{K K} \rightarrow W W \rightarrow q q \ell v\end{array}$ \\
\hline & $\begin{array}{l}\text { Bulk RS } G_{K K} \rightarrow H H \rightarrow b \bar{b} b \bar{b} \\
\text { Bulk RS } g_{K K} \rightarrow t \bar{t}\end{array}$ \\
\hline
\end{tabular}

Bulk RS $g_{K K} \rightarrow t=$
2UED / RPP

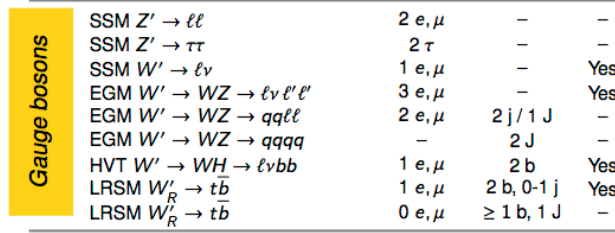

उ $\mathrm{Cl} q q q q$

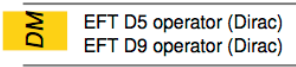

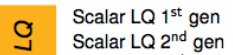
Scalar LQ $3^{\text {rd }}$ gen

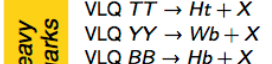
$\mathrm{VLQ} B B \rightarrow Z b+X$ $T_{5 / 3} \rightarrow W t$

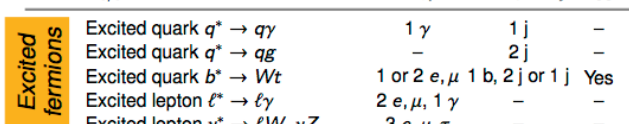

出

Excited lepton $v^{*} \rightarrow \ell W, v Z$
(n)

LSTC $a_{T} \rightarrow W \gamma$

LRSM Majorana $v$

Higgs triplet $H^{ \pm \pm} \rightarrow \ell \ell$

Higgs triplet $H^{ \pm \pm} \rightarrow \ell \tau$
Monotop (non-res prod)

Monotop (non-res prod)

Magnetic monopoles

$$
\sqrt{\mathrm{s}}=7 \mathrm{TeV} \quad \sqrt{\mathrm{s}}=8 \mathrm{TeV}
$$

$\ell, \gamma \quad$ Jets $E_{\mathrm{T}}^{\text {miss }} \int \mathcal{L} \mathrm{dt}\left[\mathrm{fb}^{-1}\right]$

\begin{tabular}{|c|c|c|}
\hline- & $\geq 1 \mathrm{j}$ & Ye \\
\hline $2 e, \mu$ & - & \\
\hline $1 e, \mu$ & $1 \mathrm{j}$ & \\
\hline $2 \mu$ (SS) & ${ }_{-}^{2 j}$ & \\
\hline$\geq 1 e, \mu$ & $\geq 2 \mathrm{j}$ & \\
\hline & $\geq 2 \mathrm{j}$ & \\
\hline $\begin{array}{c}2 e, \mu \\
2 \gamma\end{array}$ & - & \\
\hline $2 e, \mu$ & $2 \mathrm{j} / 1 \mathrm{~J}$ & \\
\hline $1 e, \mu$ & $2 \mathrm{j} / 1 \mathrm{~J}$ & \\
\hline & $4 \mathrm{~b}$ & \\
\hline \multicolumn{3}{|c|}{$\begin{array}{r}1 e, \mu \geq 1 \mathrm{~b}, \geq 1 \mathrm{~J} / 2 \mathrm{j} \text { Yes } \\
2 e, \mu \text { (SS) } \geq 1 \mathrm{~b}, \geq 1 \mathrm{j} \text { Yes }\end{array}$} \\
\hline $2 e, \mu$ & - & \\
\hline $2 \tau$ & - & \\
\hline $1 e, \mu$ & 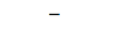 & \\
\hline $3 e, \mu$ & - & \\
\hline $2 e, \mu$ & $2 \mathrm{j} / 1 \mathrm{~J}$ & \\
\hline-2 & $2 \mathrm{~J}$ & \\
\hline $1 e, \mu$ & $2 \mathrm{~b}$ & Ye \\
\hline $1 e, \mu$ & $2 \mathrm{~b}, 0-1 \mathrm{j}$ & \\
\hline $0 e, \mu$ & $\geq 1 \mathrm{~b}, 1 \mathrm{~J}$ & \\
\hline
\end{tabular}

$\begin{array}{cccc}2 e, \mu & - & - & 20.3 \\ e, \mu \text { (SS) } & \geq 1 b, 1 j & Y_{e S} & 20\end{array}$

$\begin{array}{cccc}0 e, \mu & \geq 1 \mathrm{j} & \text { Yes } & 20.3 \\ 0 e, \mu & 1 \mathrm{~J}, \leq 1 \mathrm{j} & \text { Yes } & 20.3\end{array}$

$\begin{array}{ccc}2 e & \geq 2 \mathrm{j} & - \\ 2 \mu & \geq 2 \mathrm{j} & - \\ 1 e, \mu & \geq 1 \mathrm{~b}, \geq 3 \mathrm{j} & Y \mathrm{e}\end{array}$

$1 e, \mu \quad \geq 2 \mathrm{~b}, \geq 3 \mathrm{j}$ Yes

$1 e, \mu \quad \geq 1 \mathrm{~b}, \geq 3 \mathrm{j}$ Yes

$1, \mu \quad \geq 2 \mathrm{~b}, \geq 3 \mathrm{j}$ Yes

$2 / \geq 3$ e, $\mu \quad \geq 2 / \geq 1 \mathrm{~b}$

$2 e, \mu, 1 \gamma$

$\begin{array}{cccc}1 e, \mu, 1 \gamma & - & \text { Yes } & 20.3 \\ 2 e, \mu & 2 \mathrm{j} & - & 20.3 \\ 2 e, \mu \text { (SS) } & - & - & 20.3 \\ 3 e, \mu, \tau & - & - & 20.3 \\ 1 e, \mu & 1 \mathrm{~b} & \text { Yes } & 20.3 \\ - & - & - & 20.3 \\ - & - & - & 7.0\end{array}$

"Only a selection of the available mass limits on new states or phenomena is shown.
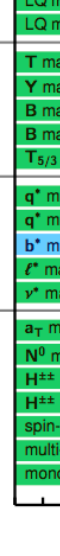

$$
10^{-1}
$$

ATLAS Preliminary

$\int \mathcal{L} d t=(4.7-20.3) \mathrm{fb}^{-1}$ $\sqrt{s}=7,8 \mathrm{TeV}$ Reference
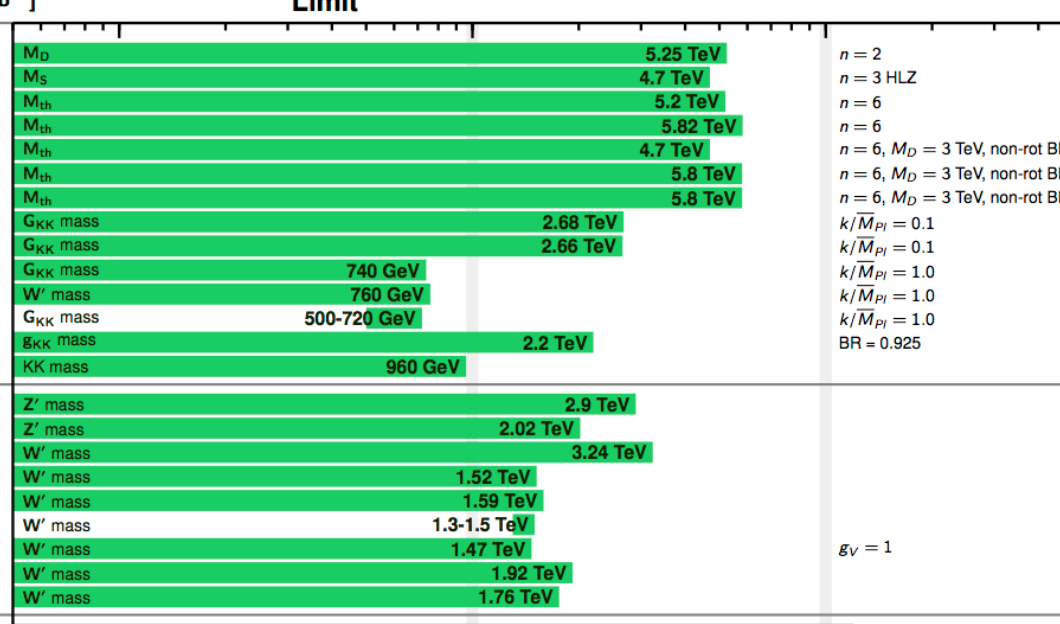

1502.01518

1407.2410
1311.2006

1407.1376

1308.4075
1405.4254

1405.4254
1503.08988

1503.08988
1405.4123

1504.05511

1409.6190
1503.04677

1503.04677
1506.00285

1505.07018 1504.04605

1405.4123

1502.07177
1407.7494

1406.4456

1409.6190
1506.00962

1506.00962
1503.08089

1410.4103 1408.0886

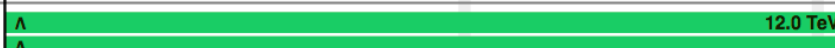

$g_{v}=1$

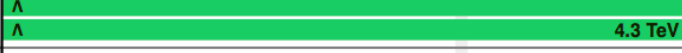

$0 \mathrm{TeV} \eta_{L L}=-1$
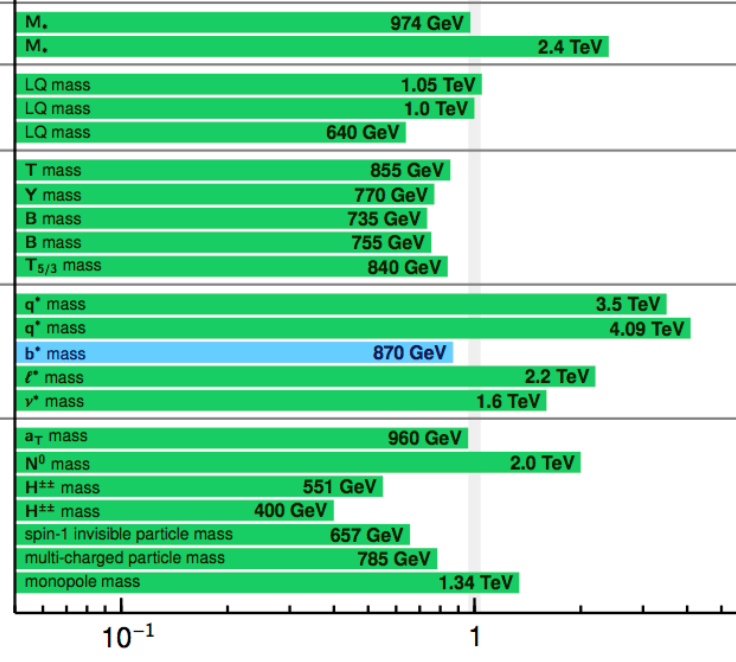
\begin{tabular}{l|l}
$\beta=1$ & Preliminary \\
Preliminary
\end{tabular} \begin{tabular}{l|l}
$\beta=1$ & $\begin{array}{l}\text { Preliminary } \\
\beta=0\end{array}$ \\
Preliminary
\end{tabular}

\begin{tabular}{l|r}
\hline$T$ in $(T, B)$ doublet & 1505.04306
\end{tabular} \begin{tabular}{l|l} 
Y in $(B, Y)$ doublet & 1505.04306 \\
\hline isospin inglet & 150505300
\end{tabular} $B$ in $(B, Y)$ doublet only $u^{*}$ and $d^{*}, \Lambda=m\left(q^{*}\right)$ \begin{tabular}{ll}
1407.1376 \\
\hline
\end{tabular} \begin{tabular}{l|l}
$\Lambda=2.2 \mathrm{TeV}$ & 1308.1364 \\
\hline
\end{tabular} $\Lambda=1.6 \mathrm{Te}$

$\begin{array}{lr}m\left(W_{R}\right)=2.4 \mathrm{TeV}, \text { no mixing } & 1407.8150 \\ & 1506.06020\end{array}$ DY production, $\mathrm{BR}\left(H_{ \pm}^{ \pm \pm} \rightarrow \ell \ell\right)=1 \quad 1412.0237$ $\begin{array}{ll}a_{\text {non-res }}=0.2 & 1411.2921 \\ & 1410.5404\end{array}$ $\begin{array}{ll}\text { DY production, }|q|=5 e & \begin{array}{l}1504.04188 \\ \text { DY production, }|g|=1 g_{D} \text {, spin } 1 / 2\end{array} \\ \text { Preliminary }\end{array}$

Mass scale [TeV]

$18 / 19$ Lomonosov-2015 


\section{... the vector-like B quarks search summary and}

\section{the lifetime limits plots}

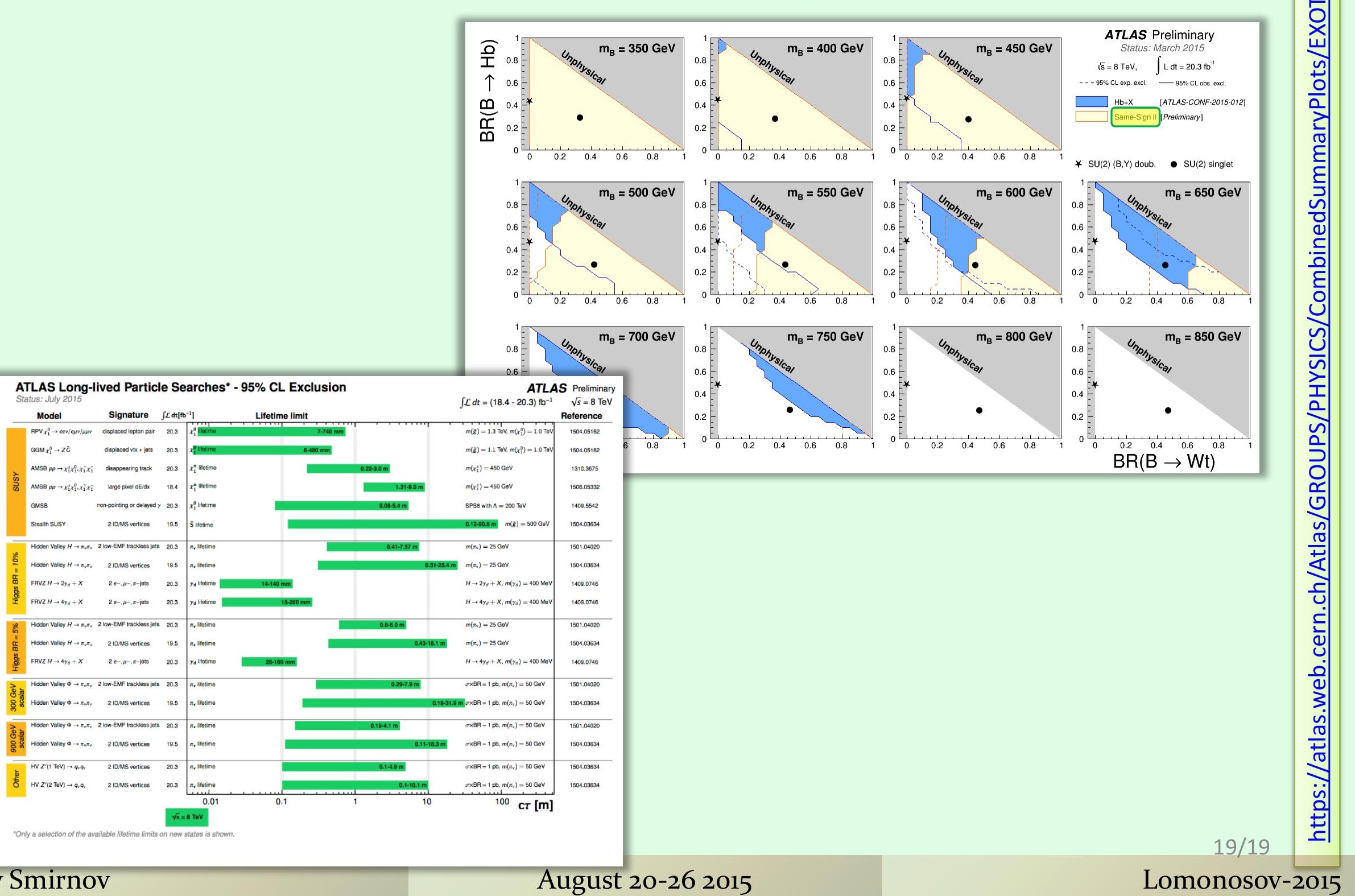




\section{THANKS!}

And stay tuned for upcoming Run-II ATLAS Exotics results! 


\section{The vector-like T quarks search summary}
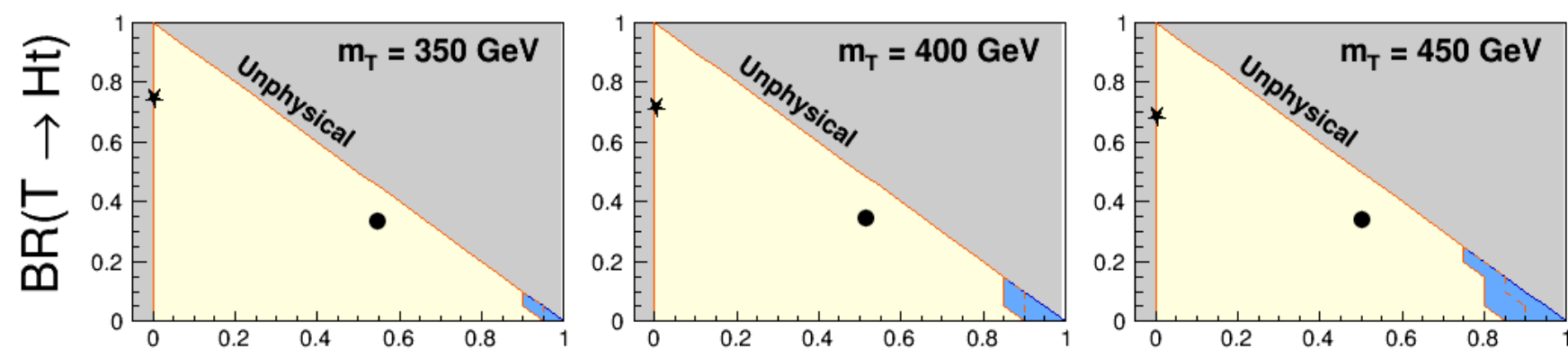

\section{ATLAS Preliminary}

Status: March 2015

$\sqrt{S}=8 \mathrm{TeV}, \quad \int L d t=20.3 \mathrm{fb}^{-1}$

- - - 95\% CL exp. excl. _ - $95 \%$ CL obs. excl.

$\square \mathrm{Ht}+\mathrm{X} \quad$ [ATLAS-CONF-2015-012]
$\square$ Same-Sign II [Preliminary]
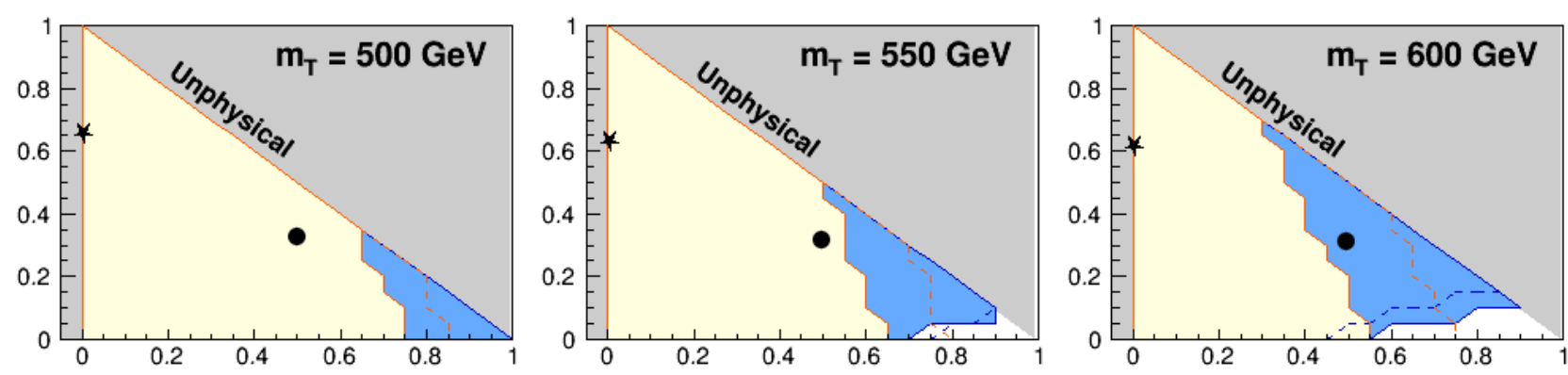

* SU(2) $(T, B)$ doub.

- SU(2) singlet
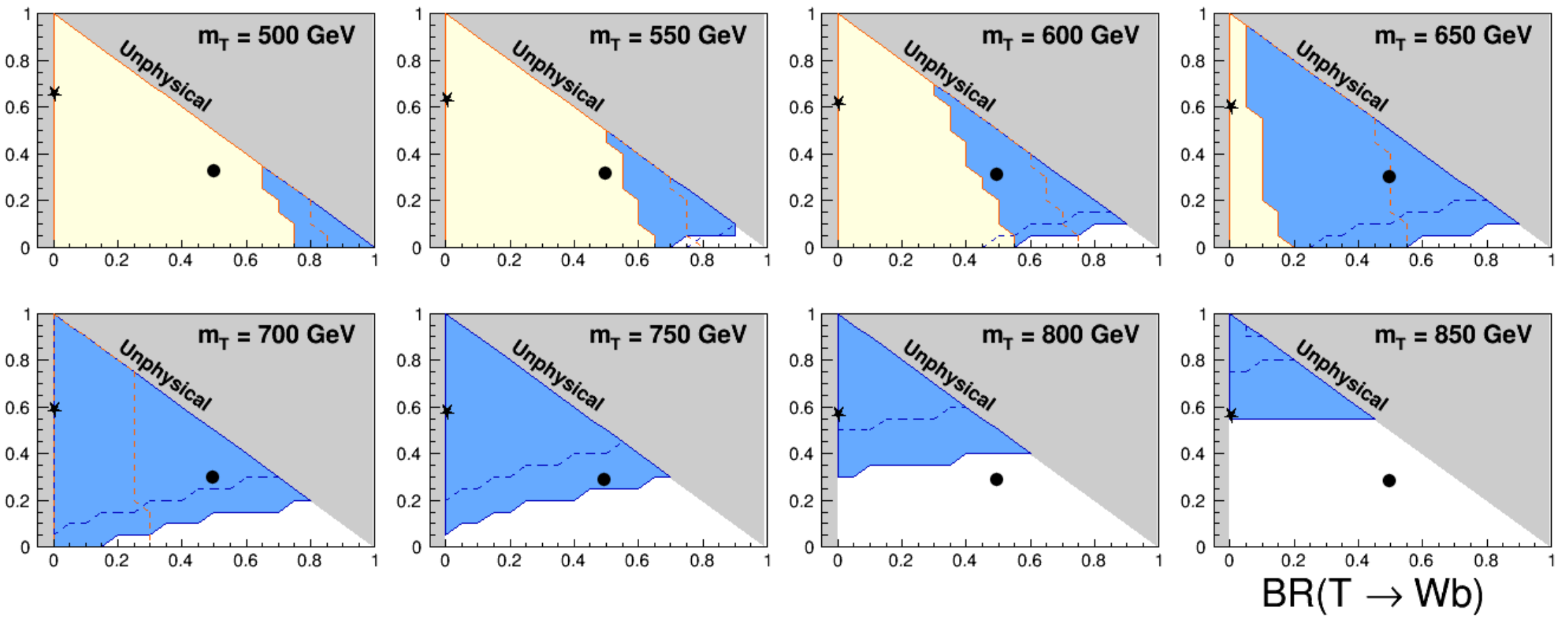